registrants contributing to oral healthcare provision in the UK, and the extent of registrant's engagement in clinical practice.

How many GDC registrants based in Europe, or further afield internationally, retain their GDC registration for purposes other than the practice of dentistry in the UK? And, what is the average commitment of GDC registrants working in the UK to the clinical care of patients? It is suggested that answers to these questions would greatly increase the value of future dental workforce planning exercises.

How could such data be collected? One solution could be findings from questions posed at the time of making the required annual statement of continuing professional development (CPD) completed to the GDC. For example: Do you anticipate providing (or contributing to) the clinical care to patients in the UK during the next 12 months? If so, how many hours a week on average do you anticipate being engaged in the care of patients? It is acknowledged that the findings from such simple questions would suffer certain limitations; however, the impact on workforce planning could be profound.

Answers to questions of the type proposed would help to protect the public and should, therefore, be seen to be an appropriate responsibility of the GDC. Assuming answers provided from individual registrants online could be collated electronically, there would be little additional burden on the Council.

N. Wilson, by email

DOI: 10.1038/.sj.bdj.2018.275

\section{Special care dentistry}

\section{Advanced care planning}

Sir, it is greatly encouraging to see the findings arising from dental teams working with memory clinics as discussed by Emanuel and Sorensen. ${ }^{1}$ Aligned with applicable publications and guidelines, ${ }^{2,3}$ the article rightly concludes that we should be encouraging prevention of dental diseases in those with early signs of cognitive decline, especially when the potential future complexity of treating these patients is considered.

Despite the importance of disease prevention, multiple studies have shown that oral diseases persist in this patient cohort ${ }^{4,5}$ which often results in the need for active intervention from dental teams. As dementia progresses, there is the potential for patients to lose the ability to express their preferences regarding treatment and to lose the capacity to consent for their treatment. Related to those processes, best-interests decisions and treatment planning can become increasingly complex and treatment provision can be associated with a greater degree of risk.

The memory clinic is likely a useful setting in which to investigate patients' experiences of dental attendance and to plan prevention approaches. For those who don't attend dental settings, I wonder if this or similar services would also be suitable forums to gather patients' preferences for their future dental treatment needs? A similar approach could be taken by dental teams for those who do attend for routine care. Though these preferences would not be fully binding without an advanced directive, an awareness of patients' past preferences could significantly assist in determination of individual patients' best interests if dental intervention becomes necessary when dementia has progressed and patients are assessed to lack the capacity to consent for treatment.

\section{A. Geddis-Regan, by email}

1. Emanuel R, Sorensen A. A study of oral health prevention behaviours for patients with early stage dementia. Br Dent J 2018; 224: 38-42.

2. Faculty of General Dental Practitioners. Dementiafriendly dentistry. London: Faculty of General Dental Practitioners, 2017

3. Pretty I A, Ellwood R P, Lo E C M et al. The Seattle Care Pathway for securing oral health in older patients. Gerodontology 2014; 31 Suppl 1: 77-87.

4. Gil-Montoya J A, Sánchez-Lara I, Carnero-Pardo C et al. Oral hygiene in the elderly with different degrees of cognitive impairment and dementia. J Am Geriatr Soc 2016; 65: 642-647.

5. Zenthöfer A, Schröder J, Cabrera T, Rammelsberg P, Hassel A J. Comparison of oral health among older people with and without dementia. Community Dent Health 2014; 31: 27-31.

DOI: $10.1038 /$ sj.bdj.2018.276

\section{Environmental issues}

\section{Dental sustainability}

Sir, the recent 'Blue Earth' programme highlighted dangers from the increasing use of plastics in disposable items. The future of marine life, the plant, and humanity appears to be at some risk.

As dentists our contribution of non-biodegradable plastics is not being addressed. The European Union Directive; Health and Safety (Sharps Instruments in Healthcare) Regulations 2013 and equivalent UK Regulations 2013 move dental practitioners away from reusable syringes towards disposable syringe-needle combinations, the rationale being to reduce the number of needle-stick injuries and hence a burden of disease, injury, and permanent or temporary impairment or handicap. Assuming hypothetical figures of 10,000 dentists administering five local anaesthetics a day over a working year of 200 days, this amounts to $1,000,000$ plastic syringes added to the plastic waste for disposal.

HTM 01-05 and other legislation introduced sealable pouches for most dental instruments. Although the need to pouch has since been reduced, again assuming 10,000 dentists using, say, 20 pouches a day, there will be in excess of 4,000,000 plastic sleeves, headrest covers and instrument pouches (most have plastic windows) to be disposed of annually, probably a conservative estimate.

Building healthy public policy, one of the five areas for action determined by The Ottawa Charter for Health Promotion, recommended the use of Health Impact Assessments (HIA) as 'means of assessing the health impacts of policies, plans and projects in diverse economic sectors'. One of the principles of this approach is 'sustainability', defined as 'a process that aims to meet the needs of the present generation without harming the ability of future generations to meet their needs'.

HIAs may be an overlooked governmental concern, but in dentistry we are not only statutorily required to carry out risk assessments and show evidence-based benefits for all procedures, but are ethically obliged and professionally responsible for doing so. Should we or could we carry out risk analyses as part of an HIA? The policy to introduce disposable syringe-needle combinations, and the demands of HTM 01-05, have been criticised for the impossibility of a true evaluation of outcomes without a valid starting database to show how many needle-stick injuries there were before the change, or the incidence of proven dental surgery induced infections prior to 2007. Without these data it may not be possible to carry out any objective based or economic evaluation, or a valid risk-assessment to see how any reduced risk of personal injury might balance against the gradual destruction of marine environment.

But we do need to seriously consider our responsibility to our planet, to future generations, and whether our actions are responsible. Perhaps the BDA could set up a commission to investigate the sustainability of policy developments, to assess the true health impacts, and to inform policy makers of the wider consequences of their decisions?

J. Aukett, by email DOI: 10.1038/sj.bdj.2018.277 